\title{
ANTECEDENTES Y VIABILIDAD DE ALTERNATIVAS EN EL ACTUAL SISTEMA-MUNDO
}

https://doi.org/10.17979/relaso.2011.1.1202

\author{
José Maria Tortosa \\ Instituto Interuniversitario de Desarrollo Social y Paz \\ Universidad de Alicante
}

Resumen: El artículo recorre las sucesivas coyunturas del sistema-mundo, desde la Guerra Fría a nuestros dias, haciendo ver que las alternativas han estado siempre presentes incluso cuando se las negaba. Con más razón lo están en la actualidad, cuando el sistema se encuentra muy alejado del equilibrio y, por tanto, necesita un menor aporte de energía para que se produzca un cambio en el mismo. Los desequilibrios actuales vienen reflejados por un lado en la aparición de los países emergentes, en la crisis de hegemonía y la existencia novedosa de países en vías de subdesarrollo y, por otro, por la acumulación de crisis interconectadas (económica, alimentaria, energética y ambiental aunque también democrática).

Palabras clave: Sistemas-mundo, crisis, alternativas, hegemonía, países emergentes, países en vías de subdesarrollo

Abstract: The paper goes though different moments of the world-system, from the Cold War to today, showing that alternatives have always been there even when they are denied. The same can be said today, when the system is very far from equilibrium, and therefore less energy input is needed to produce a change in it. The current imbalances are represented on the one hand in the appearance of the emerging countries, the crisis of hegemony and the existence of underdeveloping countries and, on the other hand, by the accumulation of interconnected crises (economic, food, energy and environmemt but also democratic).

Key words: World-systems, crisis, alternative hegemony, emerging countries, underdeveloped countries 
Hommes réels pour qui le ésespoir

Alimente le feu dévorant de l'espoir Ouvrons ensemble le dernier bourgeon de l'avenir Paul Eluard, La victoire de Guernica, 1938

La Historia acaba mostrando que habia alternativas incluso cuando se proclamaba desde el poder que no las había, según el dicho de la primera ministra Margaret Thatcher: TINA, "There Is No Alternative", no hay alternativas. Además, es posible que el TINA entonces promulgado fuese el que las ha acabado produciendo tarde o temprano.

El propósito del presente trabajo no es hacer una lista de tales alternativas que, como se ha dicho, existen, muchas están publicadas y algunas se recogen aquí en notas a pie de página. La finalidad, más bien, es, sin recrearse en "falsas esperanzas" de dudosas consecuencias (Scruton, 2010; Wallerstein, 2005a), reconocer que, en la actual coyuntura del sistema mundial, las alternativas pueden ser mucho más eficaces de lo que lo fueron en el pasado y, además, que son particularmente necesarias dado el aparente "cuello de botella" al que la proliferación de aquel TINA neoliberal llevó al sistema mundial. Para ello, se recordará brevemente el camino recorrido desde la estabilidad y las certezas de la Guerra Fria hasta el momento presente en el que la incertidumbre se ha convertido en dominante.

\section{Antecedentes}

La estabilidad del mundo de la Guerra Fría venía de dos frentes. Por un lado, tenía que ver con la pretensión de cada una de las partes: ambas creian ser inmutables y se sentian llamadas, por Dios o por la Historia, a conquistar a la otra convirtiéndola en un avatar de sí misma. Por otro lado, también tenía que ver con la relación entre las dos superpotencias capaz de asegurar la perduración del sistema de la Guerra Fría al no verse alternativa ni al cambio interno de las partes ni, tampoco, al particular tipo de relación establecida entre ellas. 


\subsection{Ambos sistemas se presentaban como si no tuviesen} alternativa interna posible. Por un lado, los sistemas supuestamente opuestos se presentaban como profundamente asentados en sus respectivas sociedades de modo que no se veía posibilidad alguna de cambio, excepto por parte de algunos visionarios como Amalrik. La distopía de Orwell en Mil novencientos ochenta y cuatro a la que se referia el citado disidente (muerto en España en 1980 en extrañas circunstancias) se podía aplicar a ambos sistemas en la medida en que parecian haber conseguido un mundo sin vuelta atrás utilizando para ello todos los medios a su alcance (Galtung, 1985), desde la violencia represiva (Goldstein, 2001) a la persuasión ideológica (Chosky y Herman, 2000) pasando por el uso del poder remunerativo.

El sistema soviético se sabia alternativa al sistema estadounidense. Es cierto que, en un primer momento, y antes de la Guerra Fría, los marxianos de la AIT, encabezados por el propio Marx, habian felicitado a Abraham Lincoln por su reelección de 1864. La clase obrera europea, decía la carta que se supone fue escrita por el propio Marx (The International Workingmen's Association, 1865), habia percibido instintivamente (sic) desde el principio que la bandera estrellada era portadora de su destino como clase. Al fin y al cabo, la Guerra Civil estadounidense acababa de terminar y la potencia hegemónica del momento, Inglaterra, había tenido motivos para estar más cerca de los Confederados y, por tanto, la AIT, anti-imperialista, tenía los suyos para estar a favor de los vencedores (Foreman, 2011). Lenin (1918), años después, en una "Carta a los obreros estadounidenses" ("americanos" según suele decirse) incidía en el papel que la clase obrera estadounidense podía tener en la lucha contra el imperialismo.

Sin embargo, la Guerra Fría iniciada después de la II Guerra Mundial (o la Gran Guerra Patria como es llamada en Rusia) cambiaría estas percepciones (y las realidades internas estadounidenses) y daría paso a la afirmación de sistemas excluyentes, llegando al famoso dicho de Kruschof ante diplomáticos occidentales que asistian a una recepción en 
Polonia: "La Historia está de nuestro lado: nosotros les enterraremos", es decir, que el sistema soviético sobreviviría al estadounidense ${ }^{1}$.

Por su parte, en los Estados Unidos predominaba (y predomina) la idea de que su modo de entender el capitalismo es el mejor y, además, el único posible. Sin embargo, no es rara la posición de los que opinan que el capitalismo (y los Estados Unidos) también están en crisis (Wallerstein, 2005b; Stiglitz, 2011).

\subsection{La relación entre los sistemas no parecia tener} alternativa viable. Por otro lado, la ausencia de alternativas (excepto la alternativa mutua, por "infiltración comunista" o "infiltración imperialista" entendida por sus respectivos agentes como "liberación") venía reforzada por lo bien trabado que quedaba el sistema internacional en el que, a primera vista, las dos superpotencias quedaban atrapadas e inmovilizadas por su "destrucción mutua asegurada" (MAD) que supondría el "invierno nuclear" que podría haber producido un enfrentamiento atómico entre los Estados Unidos y la Unión Soviética (Ehrlich, 1984). El "dilema del prisionero" que impedía el desarme daba a aquella relación la apariencia de inalterable. Pero el sistema también quedaba trabado socialmente ( estructuralmente, si se prefiere) como se muestra en el gráfico construido a partir del artículo de Johan Galtung (1971) más citado:

${ }^{1}$ La versión de la revista Time ("We will bury you!", 26 de septiembre de 1956) está todavía disponible en línea en http://www.time.com/time/magazine/article/0,9171,867329,00.html 


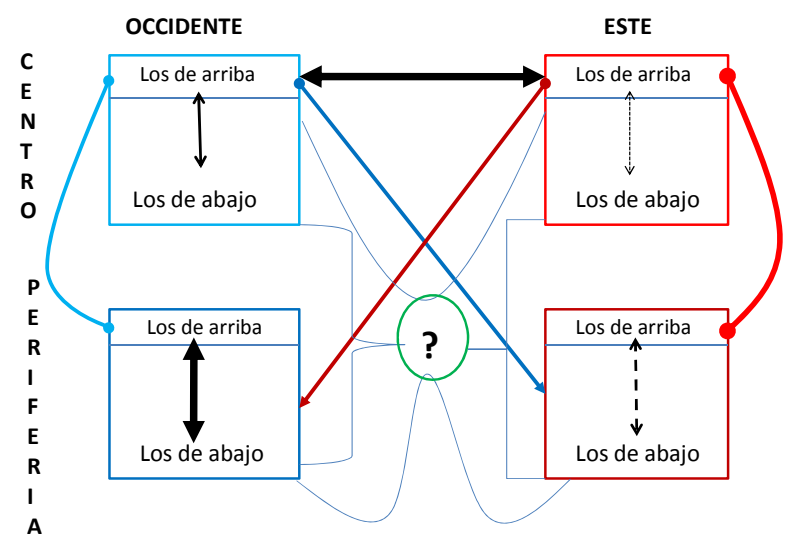

El gráfico, elaborado a partir de dichas ideas, muestra una visión simplificada del mundo de la Guerra Fría, pero no tan simplificada como para suponer que se trataba de un enfrentamiento entre dos ideologias, el "capitalismo" y el "comunismo" como los dirigentes de ambos lados se encargaban de proclamar. Cada bloque (el Este y Occidente), como se ve, estaba compuesto por países centrales (básica y respectivamente, la Unión Soviética y los Estados Unidos) y países periféricos. Dentro de cada país había y, en su caso, hay desigualdad y jerarquía. Para no entrar en disputas de escuela sobre la propiedad privada o estatal de los medios de producción, se usa aquí el vocabulario que Orwell, en su mentada novela, pone en boca del personaje Emmanuel Goldstein: "los de arriba" y "los de abajo".

Hay que insistir en que se trata de una simplificación. Entre países centrales y países periféricos habria que introducir los semiperiféricos y, obviamente y siguiendo el vocabulario del supuesto Emmanuel Goldstein de la novela de Orwell 1984, entre "los de arriba" y "los de abajo" habria que introducir "los de en medio". Y aún así seguiría siendo una simplificación, necesaria cuando no se tiene como ideal el imposible e inútil "mapa a escala $1: 1$ " del que se reía Borges, sino el de intentar comprender los rasgos básicos de una situación.

El enfrentamiento (el conflicto) era de dos tipos: el que se producía entre las respectivas élites de los países centrales 
(conflicto geopolítico por lograr la hegemonía o mantenerla, según fuese el caso) y el conflicto social entre las clases, frecuentemente de "los de arriba" contra "los de abajo" y raramente al revés, excepto en algunas contadas ocasiones en las revoluciones o intentos de revolución en los países de las respectivas periferias. Ambos enfrentamientos, cuya intensidad viene reflejada en el grosor de las diferentes flechas, daban al sistema su dinamismo. En el campo occidental está el caso de las diferentes revoluciones. En el Este, el abanico era más amplio pues iba desde los disidentes (enviados o no al Gulag o a los psiquiátricos) hasta llegar a las "primaveras" y levantamientos en Budapest (1956), Praga (1968) y, el final, en Polonia a partir del sindicato Solidarnosć y que llevaria a la "ley marcial" de Jaruzelski en 1981 que puede entenderse como "el fin del principio".

La estabilidad, en cambio, más allá de los acuerdos, de Yalta a Malta, para dividirse el mundo, venía de otro doble elemento: la alianza entre las élites de los países centrales con las élites de los países de su periferia y la "fraternal" o "solidaria" ayuda de las élites de los países centrales de un bloque a "los de abajo" del otro bloque. De alguna manera, la alternativa de "los de abajo" de la periferia de cada bloque venía determinada por "los de arriba" del país central del bloque contrario ${ }^{2}$. En cambio, y simbolizado en el gráfico con un signo de interrogación, "los proletarios de todos los países" no parecian muy unidos precisamente, siendo, por lo general, pasto de las propuestas nacionalistas de las respectivas élites, estas sí internacionalizadas. También sigue siendo la excepción la solidaridad entre países de la periferia, incluyendo a sus élites, como se ve a la hora de cambiar las reglas coloniales de los cargos en el Fondo Monetario Internacional y el Banco Mundial.

2 Un caso del sometimiento de "los de abajo" de la periferia de un bloque a los intereses de "los de arriba" del otro se encuentra descrito en primera persona en el Homenaje a Cataluña de George Orwell: la revolución en la España de la Guerra Civil de 1936 habría sido abortada por los intereses de los dirigentes soviéticos del momento. 
Es frecuente, y eso define a la respectiva escuela de pensamiento, reducir la historia de la humanidad a la historia de sus luchas o sus contradicciones o alternativas, sean contradicción entre ideas (G.W.F. Hegel), lucha de clases (K. Marx), choque de civilizaciones (S.P. Huntington) o "colapsos" o "venganzas" ecológicas (J. Diamond o J. Lovelock) que terminan cuando uno de los elementos triunfa sobre el otro. Excepto en el caso de la lucha contra la Naturaleza, que podria abocar al fin de la especie humana, todas las demás luchas no llevan necesariamente a un "fin de la historia". Mao Zedong ${ }^{3}$ lo tuvo claro, probablemente por su raíces daoístas, no por las marxistas. Sin embargo, a tenor de lo dicho, la historia de la Guerra Fría fue algo más que un enfrentamiento dicotómico, lo cual no quita para que el colapso (inesperado para muchos) del PCUS y, con él, el de la URSS y el Pacto de Varsovia entre 1989 y 1992, fuese saludado como un Fin de la Historia, según el texto de Francis Fukuyama.

El planteamiento era claro: si la historia reciente de la Humanidad ha sido, según pensaban, la historia de la lucha del "capitalismo" y el "comunismo", la desaparición de este último, en buen hegelianismo, habria significado el fin de la Historia al no tener el capitalismo alternativa alguna, ni interna ni externa. Finalmente, según se había afirmado

3 "Sobre el tratamiento correcto de las contradicciones en el seno del pueblo", publicado el 19 de junio de 1957 en Diario del Pueblo (en línea) http://www.ucm.es/info/bas/utopia/html/oet5_58.htm, acceso el 18 de julio de 2011. Obsérvese, de paso, que la única cita que hace Mao en dicho texto es a Lao Tzu, formulador, en "La regla celeste", de la idea de que las contradicciones -yin y yang- forman parte "eterna" de la realidad y, por tanto, no habría "fin de la historia". Eso no quita para que Mao afirme: "La filosofia marxista sostiene que la ley de la unidad de los contrarios es la ley fundamental del universo. Esta ley tiene validez universal, tanto para la naturaleza y la sociedad humana como para el pensamiento del hombre. Los lados opuestos de una contradicción forman una unidad y a la vez luchan entre sí, lo cual produce el movimiento y el cambio de las cosas. En todas partes existen contradicciones, pero estas tienen diverso carácter según sea la naturaleza de las cosas. En cualquier cosa concreta, la unidad de los contrarios es condicional, temporal, transitoria $\mathrm{y}$, por eso, relativa, mientras que la lucha entre los contrarios es absoluta". 
anteriormente, TINA, There Is No Alternative. Y sin embargo la hubo: otros mundos eran posibles.

\section{Coyuntura}

Hubo alternativa: otros mundos no sólo fueron posibles sino que fueron reales. Por un lado, por la aparición de nuevos actores y de alteraciones en los países centrales. Y, por otro, por la acumulación de crisis que aquel TINA (thatcherista, 1979-1990, y reaganista, 1981-1989) ha traído consigo.

2.1. Está, en efecto, la reestructuración del sistema mundial por lo menos desde tres puntos de vista: el posible colapso de los Estados Unidos, la aparición relevante de los llamados "países emergentes" (BRIC en un principio, BRICS recientemente, es decir, el Brasil, Rusia, la India, la China y Sudáfrica) y la novedad de que haya países a los que se les puede llamar "países en vias de subdesarrollo", categoria que se podría añadir a los convencionales "países en vias de desarrollo" y a "países menos adelantados".

a. El colapso de los Estados Unidos es también el colapso de lo que ha sido una constante en dicho país: "business politics", el sometimiento de la política a los intereses de los negocios en general y de las grandes empresas en particular que no es novedad ya que ya fue denunciada en 1896 en una carta atribuida al presidente Abraham Lincoln y supuestamente enviada a su amigo el coronel William F. Elkins el 21 de noviembre de 1864, cuando su Guerra Civil entraba en el último año ${ }^{4}$. Cierto que en el presente caso, "los

\footnotetext{
4 "As a result of the war, corporations have been enthroned and an era of corruption in high places will follow, and the money power of the country will endeavor to prolong its reign by working upon the prejudices of the people until all wealth is aggregated in a few hands and the Republic is destroyed. I feel at this moment more anxiety than ever before, even in the midst of war. God grant that my suspicions may prove groundless", en Archer H. Shaw, The Lincoln Encyclopedia, Nueva York, Macmillan, 1950. El texto ya se le atribuia en 1896. Menos discutible parece el siguiente: "These capitalists generally act harmoniously and in concert to fleece the people, and now that they have got into a quarrel with themselves, we are called upon to appropriate the people's money to settle the quarrel". Discurso al
} 
de arriba", de producirse tal colapso, encontrarán acomodo en un mundo "global" como muchos apparatchiki comunistas encontraron acomodo con el colapso de la URSS. Algunos, como Vladimir Putin desde la KGB en Rusia o Leszek Balcerowicz desde el Partido Obrero Unificado de Polonia comunista-, se recolocaron en la nueva situación política post-comunista. Otros, lo encontraron a través de las privatizaciones mediante un acceso privilegiado a la propiedad ahora privada de los medios de producción después de haberlos controlado como propiedad estatal, es decir, del Partido.

No se trata, obviamente, del mismo tipo de colapso que incluye desmembración de un imperio territorial como el de la extinta URSS. Aquí se trata, más bien, de la pérdida de hegemonía como Inglaterra perdió su papel hegemónico entre finales del siglo XIX y principios del XX. La historia del sistema mundial capitalista es también la historia de la lucha por la hegemonía y de los sucesivos países que la han ocupado después de que los Austrias (antes de la existencia de Estados nacionales) perdieran la que obtuvieron por ocupación territorial conquistadora. Perder la hegemonía significaria dejar de controlar las reglas del juego mundial y no poder alterarlas a conveniencia en beneficio de "los de arriba" propios y, secundaria aunque no necesariamente, del resto de la sociedad "nacional".

La crisis de hegemonía de los Estados Unidos parece clara aunque sus efectos y resultados estén por manifestarse. Iniciador de la crisis económica, con ciudadanía sometida al "masaje" contra-ideológico, consumidora insaciable de energía, entre las primeras productoras de cereales y primer (o segundo, después de la China) país emisor de $\mathrm{CO}_{2}$. La cuestión de hegemonía no se refiere tanto a su papel actual, sino al cambio que se está produciendo en el mismo.

El sistema-mundo capitalista ha conocido sucesivas hegemonías en sus más de 500 años de historia, así que, del

congreso de Illinois, enero de 1837, según Lincoln's Complete Works de 1905. 
mismo modo que los Estados Unidos sustituyeron a la Gran Bretaña como hegemón mundial (cuando "Britannia ruled the waves"), ahora podría estar sucediendo algo parecido, aunque no sea de descartar una superación de sus actuales problemas y un retorno a la hegemonía de los años 50 (que es, al fin y al cabo, el proyecto de los neoconservadores: hacer del siglo XXI un "nuevo siglo americano", es decir, estadounidense ${ }^{5}$ ). En cualquier caso, sea que se recupera, sea que es sustituida por otra potencia, sea que el sistema mundial se fragmenta en regiones más o menos estructuradas al modo de la Unión Europea, el caso que pocos niegan es que la hegemonía de los Estados Unidos está en crisis, es decir, que "ya no" es lo que era (aunque podría volver a serlo) y "todavía no" se sabe lo que pueda venir después, y más con lo maltrecha que se encuentra su economía, origen de la actual crisis económica.

Puede pensarse que "todavia" es potencia hegemónica si se atiende a la magnitud de su economia (primer Producto Interno Bruto del mundo, seguido de cerca por la China aunque no per capita), a su gasto en el sector militar (su presupuesto de "defensa" supone casi la mitad de todos los presupuestos militares del mundo, precisamente el 43 por ciento, según las estimaciones del SIPRI para 20106), al papel que todavía juega el dólar en el comercio internacional en general y en el petrolero en particular y en concreto en América Latina y en la composición de las reservas de divisas en el mundo y, last but not least, el uso sistemático que hacen sus élites de lo que su departamento de Estado llama "diplomacia pública", es decir, diseminación de información, noticias y modos de ver las cosas dirigida no a los gobiernos de otros países, sino a los ciudadanos de los mismos para que estos vean el mundo en los términos más favorables para aquellas élites.

5 Aunque inactiva, al parecer, desde 2006, sigue siendo interesante la página del Project for a New American Century: http://www.newamericancentury.org/index.html.

6 (En linea) http://milexdata.sipri.org/, acceso el 18 de julio de 2011. 
Sin embargo, Joseph Stiglitz ${ }^{7}$ identificaba siete déficits en los Estados Unidos, a saber, el déficit de valores, el déficit climático, el déficit de igualdad, el déficit de responsabilidad, el déficit comercial, el déficit fiscal y el déficit de inversión, que reducirian bastante los entusiasmos que los anteriores datos pudieran provocar y más con el problema de la suspensión de pagos de su gobierno federal, además de la de otros gobiernos locales. Que los Estados Unidos se encuentren en decadencia era reconocido, en 2010, por el 65 por ciento de los estadounidenses entrevistados y rechazado por el 31 por ciento ${ }^{8}$ Además, el gasto militar, como bien supieron potencias hegemónicas anteriores como los Austrias (y los Borbones) o Inglaterra (Kennedy, 2004), permite un cierto dominio del mundo, pero debe ser sufragado de alguna manera que, por lo general, consiste en la sobre-explotación de los propios ciudadanos (los reflejados en la "literatura picaresca" española del Siglo de Oro o en las obras de Dickens en Inglaterra) y en la explotación de las colonias. Esto último en términos de extracción de metales preciosos (a la "española"), de ocupación territorial a la inglesa o, a la estadounidense, de dominio de las reglas del juego comerciales creadas en función de los intereses de las propias élites o sufragadas mediante el endeudamiento masivo ${ }^{9}$. Más

7 Joseph Stiglitz, "Los siete déficits mortales", (Mother Jones, Noviembre/Diciembre 2008), traducido en Sin Permiso, 23 de noviembre de 2008 (en línea) http://www.sinpermiso.info/textos/index.php?id=2190.

8 NBC News/Wall Street Journal Survey, Estudio 10.805, agosto de 2010 (en linea)

http://online.wsj.com/public/resources/documents/LateAugustWSJN BCpoll.pdf, acceso el 18 de julio de 2011,

${ }^{9}$ Los datos son de difícil verificación, pero el "reloj" que marca la deuda pública estadounidense (http://www.brillig.com/debt_clock ) ya alcanzó en enero de 2011 los 14 billones de dólares que viene a ser la cifra de su Producto Interno Bruto y más de un tercio del total de la deuda pública

mundial

(http://www.economist.com/content/global_debt_clock ). El Center on Budget and Policy Priorities hace un cálculo diferente: en fechas parecidas, alcanzaría en torno al 70 por ciento del PIB y la mitad de dicha deuda estaría originada por los recortes fiscales iniciados bajo el segundo Bush y los gastos militares. Talking Points Memo, "Bush 
bien, y en términos comparativos, el exceso de gasto militar es como una "supernova" que anuncia el final de la estrella y su paso a "enana blanca". De momento, los Estados Unidos, en el terreno político, han ido sufriendo pequeñas derrotas, tal vez poco importantes en sí mismas como en la tortura china de "los mil cortes" (Wallerstein, 2005c), pero cuya acumulación podría acelerar la decadencia, vaya o no vaya a producirse, pues nada hay que proporcione certeza al pronóstico en cualquiera de las dos hipótesis.

Pero, de nuevo, eso es una crisis y, como las anteriores crisis de hegemonía conocidas, va acompañada de inestabilidad estructural y "la intensificación de la competencia interestatal e interempresarial; la escalada de los conflictos sociales; y el surgimiento intersticial de nuevas configuraciones de poder" que es lo que se concluye de un análisis comparado de las sucesivas crisis de hegemonia (Arrighi y Silver, 2001: 6). Traducido al tema presente, la crisis de hegemonía permite acciones y decisiones de rebeldia alternativa que habrian sido impensables en los momentos en que la potencia hegemónica estaba en su cénit. La diferencia es que ahora no tiene la "fraternal" ayuda del extinto bloque soviético, aunque comienzan a aparecer nuevas fuentes de ayuda contrahegemónica: los BRIC.

b. Los paises emergentes. Como en "El pastor y el lobo", la fábula de Esopo, la decadencia de los Estados Unidos ha sido anunciada repetidas veces (incluida la de Kruschof) y es lógico que los nuevos anuncios sean recibidos con escepticismo como en la fábula. Pero también la fábula termina con la llegada efectiva del lobo aunque nadie se lo crea. Este parece ser el caso ${ }^{10}$, abonado por la presencia del grupo de los llamados países emergentes, el Brasil, Rusia, la India, la China a los que se les ha añadido Sudáfrica para

Policies Dominant Cause of National Debt", 25 de mayo de 2011 (en linea) http://tpmdc.talkingpointsmemo.com/2011/05/chart-bushpolicies-dominant-cause-of-debt.php?ref=fpa.

10 Ver el número especial de Foreign Policy, "American decline. This time is real", $\quad$ enero/febrero 2011 (en línea) http://www.zinio.com/reader.jsp?issn=0015-7228, acceso el 18 de julio de 2011. 
componer el grupo de los BRICS, con reuniones periódicas para coordinar políticas económicas (y probablemente monetarias), relaciones exteriores $\mathrm{y}$, tarde o temprano, sistema militar.

Se trata de un grupo muy heterogéneo en antecedentes y situaciones internas: la pobreza en la China no es la de la India, la desigualdad del Brasil no es la de Rusia y el impacto de la crisis de 2008 no ha sido el mismo en la China y la India por un lado y en el Brasil y Rusia por otro ${ }^{11}$ y así sucesivamente. Pero todos los emergentes tienen un territorio inmenso, una economía que ha mantenido tasas de crecimiento positivas (e incluso comparativamente altas) en la crisis económica actual, ambiciones de, por lo menos, ser potencia de ámbito local, regional, y tres de ellas disponen de armas nucleares aunque las otras dos (el Brasil y Sudáfrica) han tenido atisbos de iniciar la carrera atómica. Además, sobre todo la China tiene un superávit comercial que le permite ser el primer tenedor de Bonos del Tesoro estadounidenses a pesar de ser "país de ingresos medianos altos" según la peculiar clasificación del Banco Mundial ya citada.

El resultado de haber aunado esfuerzos es que han podido forzar decisiones de los países centrales años atrás impensables. Aunque el G-8 sigue existiendo, estos han tenido que cooptar al G-20, y su papel en el Fondo Monetario Internacional y en el Banco Mundial ya no es el que era y los no-miembros del Consejo de Seguridad de Naciones Unidas pueden maniobrar para lograr un puesto de miembro permanente. Está por ver si esta emergencia lleva a que uno de ellos (tal vez la China) sustituya a los Estados Unidos como potencia hegemónica o a que el mundo se fragmente en torno a estos 5 centros añadidos al de los Estados Unidos, el Japón y Europa.

11 Son los países centrales los peor parados mientras la tónica de los periféricos es positiva, y muy positiva en algunos emergentes (Fondo Monetario Internacional, 2011) 
Sí tendría que estar claro, a tenor de lo dicho, que el vocabulario "geográfico" ("Norte-Sur", "Sur Global”, "Sur-Sur") puede ser movilizador, pero su valor analítico es muy discutible $\mathrm{y}$, probablemente, buscar alternativas desde aquella perspectiva puede llevar a diagnósticos equivocados $\mathrm{y}$, consiguientemente, a terapias igualmente erradas. En cambio, el papel creciente en el sistema mundial por parte de los emergentes permite nuevas alianzas alternativas al sometimiento al "Norte" en general y a los Estados Unidos en particular: vuelve a estar presente la "fraternal ayuda" que hubo durante la Guerra Fría esta vez por parte de potencias, generalmente los BRICS, que no necesitan cubrir los intereses de sus clases dominantes y/o dirigentes con mantos ideológicos (Michel y Beuret, 2009; CEPAL, 2010; Chomsky, 2010).

c. Además de eso, está la aparición de los países en vías de subdesarrollo, una nueva categoría para clasificar países. Manfred Max-Neef, economista chileno, publicó con Philip Smith un libro (Smith y Max-Neef, 2011) con un capitulo titulado "Estados Unidos, un país en vias de subdesarrollo". Es discutible tal afirmación aunque no son los únicos en hacerla (Galtung, 2010). No es tan discutible, en cambio, que su hegemonía está en crisis, como se acaba de ver, aunque no puede descartarse que el proyecto neoconservador triunfe y el país (es decir sus élites) vuelva a ser hegemónico. Lo que, en cambio, es menos discutible es que la situación de algunos países pueda tildarse de propia de "países en vias de subdesarrollo". Es el caso de Grecia, Portugal e Irlanda. Y, probablemente, España, y la lista no es completa ya que ha tenido como candidatos también a Italia y a Bélgica, aunque por motivos diferentes. Un rápido recorrido por las características del subdesarrollo teniendo en mente la situación de los PIGS (y la de los Estados Unidos) puede resultar instructivo:

- Un país subdesarrollado suele tener problemas con su deuda externa, problemas que llevan a crisis de deuda en las que interviene el Fondo Monetario Internacional con sus "condicionalidades". Esa deuda ha sido 
generada por mala gestión interna, pero también por presiones externas que van desde el exceso de liquidez en bancos extranjeros a la generación de dependencia política planificada como describe el libro de John Perkins (2005), Confesiones de un sicario económico. Las presiones para las "condicionalidades" vienen de fuera $(\mathrm{y}$ por eso no se aplican a los Estados Unidos, que todavia dispone de otras medidas para afrontarla desde su hegemonia) aunque con apoyo interno.

- Un país subdesarrollado, sometido a dichas "condicionalidades", tiene que reducir su gasto público, mejorar su balanza comercial (exportar más, importar menos) y contener la inflación. Los efectos sociales de dichas políticas económicas, cuando no economicistas, son devastadores. América Latina los conoció al gestionar su deuda.

- Un país subdesarrollado suele ser un país en el que se disparan los índices de percepción de la corrupción de Transparencia Internacional y en el que las encuestas de Gallup muestran una generalización de comportamientos corruptos (sobornos, "mordidas", comisiones ilegales). El prestigio de su clase politica, en consecuencia, es muy bajo.

- Un país subdesarrollado sufre una sangría continua en su fuerza de trabajo: los más dispuestos se van y, en particular, se produce una fuga de cerebros, de jóvenes preparados que se trasladan a donde esperan obtener empleo de acuerdo con su titulación. Es decir: la inversión social en educación en el país es disfrutada por otros países.

- Un país subdesarrollado suele tener un modelo económico que puede llamarse "comodón", es decir, se especializa en sectores que tienen poco impacto en otros sectores y con relativamente escaso valor añadido. Por ejemplo, se dedican a lo que en América Latina se llama extractivismo (mineria, petróleo, materias primas en general) o a lo más parecido al mismo que es el turismo de "sol y playas". 
- Un país subdesarrollado, como efecto de dicho modelo, es particularmente sensible y vulnerable hacia fuera por las fluctuaciones internacionales o medioambientales. Hacia dentro, lo es por aumentos de la desigualdad social que se convierten en freno al crecimiento económico que sería necesario para generar empleo en una economía que no sea sumergida (los "informales" en América Latina) y cuyos empleos sean mayoritariamente no precarios.

- Un país subdesarrollado presenta una presión fiscal muy baja, razón por la cual el gasto social es igualmente bajo. En general, los ricos no sólo cotizan menos, comparativamente hablando, sino que si se reduce algún impuesto, resulta ser el que mejor satisfaga la codicia y poder de los ricos, por aquello reaganista de que disminuir los impuestos (de los ricos) genera mayor recaudación fiscal (decisión, que se mantiene hasta ahora, legitimada mediante la curva de Laffer).

- Un país subdesarrollado suele tener un sector público sanitario y educativo de poca calidad, no universal. Como hay que reducir gastos para pagar la deuda y compensar lo que se pierde por bajar impuestos (como el de sucesiones), esos gastos se compensan con una reducción del presupuesto de la sanidad y de la educación y su consiguiente privatización, supresión de su gratuidad o introducción del copago.

La Historia, efectivamente, no ha terminado y países que estuvieron en el centro, como los PIGS, y no solo ellos, pueden estar camino de la periferia, "en vías de subdesarrollo", alterando el sistema mundial en términos que incluyen la posibilidad de profundos cambios en/de la Unión Europea.

\subsection{La acumulación de crisis multiplica los problemas} que exigen alternativas. Pero es que lo dicho ha de situarse en el contexto de la crisis económica iniciada en 2007 (se arrastraba de mucho antes) y que comenzó a ser percibida en 2008. Crisis, como situación en la que "ya no", pero "todavía 
no": algo ha terminado, pero todavía no está claro qué lo va a sustituir.

A diferencia de la crisis económico-financiera de 1929-1939, en la actual se añaden otras crisis, anteriores o agudizadas por la misma que inciden en la dificultad en superarla formando lo que puede llamarse una "crisis global" (CG) que va a influir de manera particular en la cuestión de las alternativas: primero porque ha llevado al sistema lejos del equilibrio $\mathrm{y}$, por tanto, precisa de menos energía para producir un cambio en el mismo, y, segundo, porque aparecen nuevos campos en los que se puede intervenir sabiendo que, si están interconectados como problemas, también pueden estar interconectadas sus soluciones.

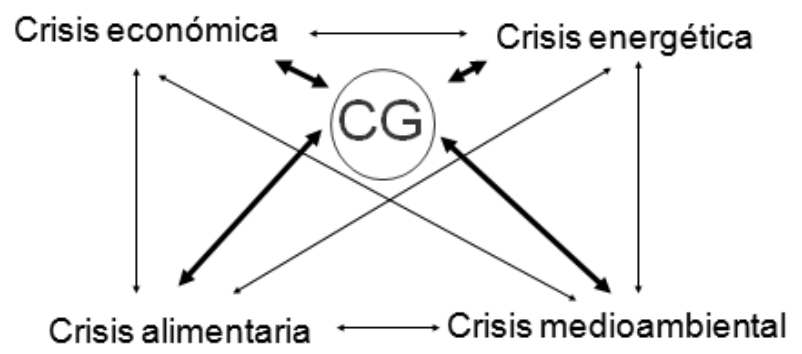

a. La crisis económica es la más visible desde la perspectiva de los países centrales, en particular, los Estados Unidos y, como se ha dicho, la de los países en vías de subdesarrollo. Entre las que forman este grupo de crisis diversas, no ha sido la primera en producirse, aunque sí la más comentada, pero el caso es que el estado en que se encontraba la economía mundial antes de 2007 ya no es el actual, mientras que no se sabe bien, dígase lo que se diga, qué es lo que puede venir ni cuándo. En este epígrafe, el problema se originó en los Estados Unidos, financiarizados, desregulados, hiperconsumistas, contaminadores, endeudados e inmersos en varias burbujas especulativas, una de ellas la inmobiliaria, pero también la financiera, la alimentaria y, periódicamente, la energética.

El efecto de lo que primero fue un problema bancario, después financiero y finalmente económico, ha sido, por lo menos hasta 2010, una contracción brusca de la economía mundial, un descenso del comercio internacional y una 
reducción del Producto Interno Bruto de numerosos países, sobre todo países centrales, ligados a la economía estadounidense, faltos de reservas $y$, como en el caso español, con sus propias burbujas especulativas, en este caso inmobiliarias. Fruto de este "decrecimiento" ha sido el aumento del desempleo, la reducción del consumo y los diversos déficits públicos con las consiguientes discusiones sobre el modo de afrontarlos (reducir el gasto o aumentar los impuestos, ambos nefastos para la crisis), siendo los más afectados los más vulnerables (sexo, edad, estatus...) de cada sociedad.

Es pronto para saber si la crisis va a ser, en este campo, tan profunda como se pensó en un principio, cuando se habló (y en voces tan poco sospechosas como las del presidente Sarkozy) de "refundar el capitalismo", es decir, es pronto para saber si el sistema mundial "ya no" es estrictamente capitalista, pero "todavía no" es algo diferente que se desconoce. Es posible que la depresión (que no recesión) sea duradera, pero sin cambiar en mucho el funcionamiento del sistema mundial, sus reglas del juego y su estructura. $O$ incluso sería posible, y también hay indicios en esa dirección, que el sistema, con su "lucha de clases desde arriba", se refuerce en su jerarquía de clases aunque haya cambios en los países que componen el centro, pasando de G-8 al G-20, eso sí, mediante cooptación de los "países emergentes" y otros intermedios por parte de los centrales. Eso es precisamente una crisis. Pero también es cierto que hay alternativas.

b. La crisis energética (un mundo que "ya no" es petrolero, pero que "todavía no" ha dejado de serlo) entra aquí desde varios puntos de vista. En primer lugar, por su impacto en la crisis económica: un aumento del precio de los carburantes supondria un peso más para la mayoria de países centrales aunque las "repúblicas petroleras" puedan sentir coyunturalmente el espejismo de la abundancia, o más bien la maldición de la abundancia (Acosta, 2009).

En segundo lugar, porque algunos países ya se encuentran en el "pico del petróleo" y es posible que el mundo esté cercano al punto en que el consumo ya se hace a costa de las 
reservas sin que los nuevos descubrimientos lo compensen. Es dificil de establecerlo con un minimo de seguridad ya que es habitual que países y empresas petroleras "maquillen" sus datos tanto por exceso como por defecto, no pudiéndose, entonces, establecer realmente de qué reservas disponen y de qué tipo de combustible (gas, petróleo) . Alberto Acosta, que fue ministro de Energía y Minas en el Ecuador, lo reconoce abiertamente para su país, no excepcional en este epígrafe, cuando dice que en otros tiempos

"[...] el manejo de las reservas petroleras fue manipulado sistemáticamente. Así, para justificar mayores tasas de extracción de crudo con el fin de atender el servicio de la deuda, se inflaban las reservas mientras que para tomar medidas favorables al capital externo, se presentaban cifras de reservas a la baja" (Acosta, 2011: 48).

Además. si los países petroleros carecen de medios "militares" para proteger lo que les queda, la necesidad de manipular los propios datos es todavía mayor visto el interés explícito de las superpotencias de garantizarse el acceso a esa energía. Con el incremento del papel geoestratégico del petróleo y el aumento de empresas petroleras estatales, el dato adquiere no sólo un significado económico sino también político hacia dentro del país, pero igualmente hacia el exterior.

$\mathrm{Y}$ en tercer lugar, hay crisis energética porque la energía en general y el petróleo en particular están imponiendo una transición energética en la que, de nuevo, lo viejo ya ha muerto, pero lo nuevo todavía está por nacer (Friedrichs, 2010), con la particularidad del papel que juegan las nuevas fuentes de energía y los problemas que genera cada una de ellas, desde el biodiésel y la crisis alimentaria de la que se habla a continuación hasta el retorno al carbón y su papel en la crisis medioambiental de la que se hablará después. Todo ello sin olvidar los problemas con la energía nuclear y la energia necesaria para construir fuentes de energía renovables (eólica y fotovoltaica).

La alternativa que se pretendió fuese la energía nuclear, tiene cada vez más problemas, por lo menos desde el desastre de Fukushima que ha hecho revisar politicas pro-nucleares 
como la inicial de Angela Merkel, ya abandonada. El carbón es problemático por razones medioambientales. Quedan las renovables, pero su aplicación no parece pueda alcanzar los niveles de prestación que tuvo el petróleo y derivados, así que sí tienen sentido comenzar a imaginar el "fin del mundo que hemos conocido".

c. La crisis alimentaria, también anterior a la económica, pero no independiente de ella, es fruto de los desajustes entre oferta y demanda de alimentos, además de resultado de una particular burbuja especulativa que hizo dispararse los precios en 2007 y ha alcanzado cifras preocupantes en 2011. Por cuestiones medioambientales de las que se hablará de inmediato, la producción de alimentos, las cosechas, se redujeron en el mundo al tiempo que una parte de ellos (cereales), reflejando la crisis del petróleo, se detraía del consumo para dedicarlo al biodiesel.

Simultáneamente, el acceso de importantes capas de la población mundial a situaciones de mayor capacidad de compra (por ejemplo, las clases medias de la China y la India, menos salpicadas por los efectos de la crisis económica), hizo aumentar la demanda que, acelerada, como se ha dicho, por su propia burbuja especulativa, hizo que los precios mundiales se disparasen. El índice mundial de precios de los alimentos se publica y actualiza por la $\mathrm{FAO}^{12}$. Según dicha fuente, el primer cuatrimestre de 2011 habría tenido los mayores incrementos en los precios desde 2007. El Banco Mundial también publica su "Alerta sobre precios de los alimentos" 13 con conclusiones semejantes aunque a partir de datos que difieren de los de la FAO. Las perspectivas para los próximos años son sombrias según un estudio de Oxfam Internacional (Bailey, 2011). El efecto está siendo dejar a capas importantes de la población mundial sobre todo de la periferia al margen del acceso a los alimentos (Brown, 2011) y eso se llama hambre.

12 En http://www.fao.org/worldfoodsituation/en/

13 http://www.bancomundial.org/temas/preciosalimentos/alertasobre-precios-2011.htm 
Con menor capacidad de producción para el autoconsumo y con menguada capacidad de compra de alimentos (por falta de recursos y/o por incrementos de los precios) el hambre tenía que aumentar, y ha aumentado, probablemente por encima de los 900 millones que calculaba la FAO para 2010. Es probable que aquella estimación de 925 millones de hambrientos se hiciese a partir de la relativa estabilidad en la aceleración de los precios que se produjo a mitad de 2010. E1 año anterior, en cambio, con incrementos más fuertes, dio como resultado una estimación de 1.020 millones de hambrientos a escala mundial y la estimación más probable para 2011 es que se vuelva a los mil millones de hambrientos.

d. La crisis medioambiental también tenía su propia lógica. Sin embargo, se agudiza en la medida en que gobiernos y ciudadanos atribuyen mayor importancia a la crisis económica que a las cuestiones ambientales y en la medida en que las crisis energética y alimentaria llevan a formas de producción y consumo no necesariamente más respetuosas del medio ambiente. Tenga o no tenga que ver con el evidente aumento de las emisiones de $\mathrm{CO}_{2}$ a escala mundial, que en 2010 se habria alcanzado un nivel récord en dichas emisiones según la Agencia Internacional de la Energía ${ }^{14}$, lleve o no lleve a un cambio climático y produzca o no produzca un aumento irreversible de las temperaturas medias en la superficie del Planeta (calentamiento global), el hecho es que dichas temperaturas se han incrementado en los últimos años produciendo efectos en el abastecimiento de agua y en las cosechas que tienen que ver con la crisis alimentaria (Welzer, 2011).

De manera simultánea, ha crecido la preocupación por los recursos no renovables del Planeta sin que los gobiernos, reunidos en sucesivas Cumbres, hayan acordado algo más importante que volverse a reunir y sin que el riesgo de desaparición de la especie a medio o largo plazo haya sido afrontado o descartado (Lovelock, 2009). Tampoco los

${ }^{14}$ Agencia Internacional de la Energía, nota de 30 de mayo de 2011 (en linea) http://www.iea.org/index_info.asp?id=1959 
ciudadanos, en su conjunto, parecen dispuestos a enfrentarse al problema y, a lo más, practican la "teoria del gorrón", es decir, esperan que los demás hagan lo que ellos no piensan hacer, pero beneficiándose de los efectos de lo que hacen los otros de manera más respetuosa con el medio ambiente y, en consecuencia, con la especie humana.

e. La crisis democrática (Vidal-Beneyto, 2010) se añade a la crisis global como efecto de la misma y teniendo un claro impacto en ella hasta que haya quien ve que la superación de la crisis democrática forma parte de la superación de la crisis económica (Touraine, 2011). No se alude solamente al hecho de que la calidad democrática de los sistemas politicos existentes deja mucho que desear tanto desde el punto de vista de los encuestados como desde la percepción de algunos analistas. Tampoco se reduce a la proliferación de "Estados fallidos", vocabulario siempre discutible, pero cuya discusión no oculta el hecho de la creciente debilidad de muchos Estados para afrontar lo que, desde Max Weber, se ha dado como constitutivo del Estado. Mucho menos tiene que circunscribirse a que el número de "democracias electorales" se ha reducido a 116 en 2009 después del máximo de 123 logrado en 2006 o, si se prefiere, que el número de "democracias plenas", según el indice de The Economist15, habría pasado de 30 en 2008 a 26 en 2010.

La crisis democrática se refiere, sobre todo, a la progresiva alienación de los ciudadanos respecto a sus respectivos sistemas politicos a los que consideran poblados de ineptos y corruptos que los convierten en ineficaces y hasta inútiles, sintiéndose, en consecuencia alienados, enajenados, extrañados de dicho sistema o incluso "indignados" ("aganaktismenoi" en Grecia) por el mismo como ha quedado patente en el movimiento "15M", "Democracia real, ya" o "Spanish revolution" y, antes, en la "primavera árabe" o en Wisconsin sobre los recortes en los derechos sindicales, con interesantes antecedentes en los "piqueteros" argentinos y en

15 The Economist, Democracy index 2010, The Economist Intelligence Unit (en linea) http://graphics.eiu.com/PDF/Democracy_Index_2010_web.pdf. 
los "forajidos" ecuatorianos, interesantes incluso por cómo terminaron: los primeros, desde un "que se vayan todos" a que todos se quedaran; los otros, los ecuatorianos, con una "revolución ciudadana" cada vez menos revolución y menos ciudadana. Pero, también, antes de los "indignados", estuvo la revuelta en Islandia pidiendo el enjuiciamiento de políticos $\mathrm{y}$ banqueros responsables de la crisis del país $\mathrm{y}$ consiguiéndolo en algún caso.

La crisis democrática también tiene que ver con la incapacidad demostrada de muchos gobernantes de afrontar con coherencia los efectos de la crisis económica y paliar los de la crisis alimentaria en su caso y, en general, de la energética. En el primer caso, son constatables la idas y venidas y la falta de consenso, muchas veces por motivos electoralistas, con grave riesgo para la economía.

Para algunos autores, y parece que con razón, forma parte también de esta crisis de las democracias el hecho de que, en muchos países, la "democracia de la opinión" está siendo sustituida por una especie de "democracia de la emoción" en la que el dato, el razonamiento, la propuesta y las politicas son sustituidas por la fobia, la exaltación, hasta la histeria, con chovinismo, totemismo a través del equipo de fútbol, "supremacismo", tribalismo, racismo, en muchos casos provocado o, por lo menos, fomentado por algunos medios de comunicación.

Las democracias también estuvieron en crisis en la anterior Gran Crisis, la de 1929-1939. Aquella coyuntura produjo numerosos movimientos alternativos (como la vuelta a la Naturaleza de los Wandervögel -pájraros errantes- alemanes), polarizó los sistemas políticos... y generó el auge de "cirujanos de hierro" que cortaron por lo sano, dejaron la desacreditada democracia y su "partidocracia" a un lado y se lanzaron a operaciones populistas y guerreras que fueron desde el nazismo a los distintos fascismos y autoritarismos de los años 40.

La Historia no tiene por qué repetirse ni en comedia ni en tragedia, pero eso no quita la oportunidad de plantear ese 
elemento particular de esta crisis, por otro lado ya visible en numerosos Estados del mundo como se deduce del aumento del hambre en los países centrales, más afectados por la crisis económica que la mayoría de países emergentes y algunos periféricos. Poco preparados para afrontar esta nueva situación, amplias capas sociales de los países centrales caen en la xenofobia y el racismo, favorecido por la crisis global y fomentado por políticos oportunistas.

f. Como conclusión puede decirse que nos encontramos ante una crisis general o global no sólo en el sentido de planetaria, aunque sus efectos sean desiguales en el centro, la periferia y los emergentes, sino, sobre todo, porque cubre muchos aspectos. Tiene elementos en común con otras acaecidas con anterioridad en ese funcionamiento cíclico que parece tener la economía mundial, por ejemplo, a través de los ciclos largos llamados de Kondratiev. Sin embargo presenta características que le son propias. Todo ello sin caer en la impersonalización: esta crisis, como las demás, es resultado de acciones humanas de seres humanos concretos que, o las provocan directamente, o producen efectos perversos al combinarse entre sí.

Pueden buscarse puntos de semejanza con la crisis de 19291939. Algunas decisiones económicas que llevaron a ella se han vuelto a repetir, en particular una vez las autoridades económicas estadounidenses se desdijeron de medidas que se habian tomado respecto a los bancos, con el objetivo de que no se reprodujese. Y algunas creencias también han podido ser comunes, como podría ser el caso de la confianza en que los mercados se autorregulan, en que la mejor política económica es la inexistente y que cualquier restricción al juego de la oferta y la demanda no es la solución sino la causa del problema. Lo mismo puede decirse de la financiarización de la economía para lograr la acumulación de beneficio, motor del sistema, aunque entonces no se dieron, como ahora, empresas "too big to fail".

También la crisis del 29 se produjo en un momento en que la potencia hegemónica de aquel momento, a saber, Inglaterra, se encontraba en crisis terminal que no quedaría 
definitivamente superada por la estadounidense hasta mitades de los años 40, cuando la política interestatal (ONU), económica (Banco Mundial, Fondo Monetario Internacional) y militar (OTAN, ANZUS, Tratado de Rio) se organizaron en torno a los intereses de las élites estadounidenses. Las élites inglesas encontraron en la "special relationship" un modo de mantenerse cerca del nuevo hegemón, pero fue obvio que ya no era cierto que "Britannia rules de waves".

Si los cambios de hegemonía mundial han sido precedidos por guerras entre las potencias centrales, guerras de las que emergía la nueva potencia hegemónica, el paso de Inglaterra a los Estados Unidos siguió la regla y la crisis del 29 se situó entre las dos guerras "mundiales" (la del 14 y la del 39), estando por ver si, ahora, la crisis de hegemonía estadounidense lleva a una guerra de parecidas características. Para algunos autores, el elemento semejante (por ejemplo, con lo que fue, simbólicamente, la Armada Invencible, es decir, el gran despliegue militar que realmente significa el fin de un imperio) podrian haber sido las guerras de Oriente Medio, de Irak a Afganistán. Cierto que no son guerras entre países centrales, pero sí son guerras en las que, como es el caso de Afganistán, desde Alejandro Magno al imperio ruso, pasando por el británico, las potencias han fracasado con consecuencias negativas para su posición en el mundo. "Afganistán, tumba de imperios". Lo que dice el pasado puede resumirse en esta tabla16:

16 Aunque hay autores, como Modelski, que discuten si fue España o Portugal la potencia hegemónica de la época, se trata de un anacronismo: los que detentaban la hegemonía en ese momento no eran los Estados o las "naciones" sino los reyes, en concreto, los de la casa de Austria. El nacionalismo y el Estado-nación son invenciones posteriores. 


\begin{tabular}{|l|l|l|}
\hline $\begin{array}{l}\text { Guerra } \\
\text { “mundial” }\end{array}$ & Potencia hegemónica & Decadencia \\
\hline $1494-1516$ & Casa de Austria, 1516-1540 & $1540-1580$ \\
\hline $1688-1713$ & Inglaterra, 1714-1740 & $1740-1792$ \\
\hline $1792-1815$ & Inglaterra, 1815-1850 & $1850-1914$ \\
\hline $1914-1945$ & Estados Unidos, 1945-1973 & $1973-$ \\
\hline
\end{tabular}

Modificado a partir de George Modelski, Long Cycles in World Politics, Seattle, University of Washington Press, 1987.

Sin embargo, las diferencias son igualmente marcadas. En 1929 se vivía todavia en un mundo de petróleo abundante y relativamente barato (hasta la crisis de 1973), lejos de las dudas actuales sobre el "pico del petróleo" y con acceso al mismo con suficiente facilidad como para no convertirlo en una prioridad geoestratégica que no llegaria, prácticamente, hasta la "doctrina Carter" en los años 80 del siglo $\mathrm{XX}^{17}$. Por otro lado, y a pesar de las penurias que acompañarian a la crisis, se iba a seguir pensando, durante mucho tiempo, que la producción agrícola en el mundo era suficiente para alimentar a toda la población mundial presente y futura, con un cierto descrédito de las ideas malthusianas ${ }^{18}$. No sólo: la producción podía aumentarse, se pensaba, gracias a la investigación agrícola y la "revolución verde". Finalmente, las preocupaciones medioambientales estaban ausentes en las agendas de los gobernantes y las ideas sobre "los limites del crecimiento" no aparecerian hasta 1972.

17 Fue formulada en su "State of the Union Address 1980", disponible en la Jimmy Carter Library (en línea) http://www.jimmycarterlibrary.gov/documents/speeches/su80jec.pht ml. El petróleo ya había sido objeto de maniobras internacionales como fue el "Big Game" en Oriente Medio por parte de los países centrales a la caída del Imperio Otomano.

18 En la actualidad, de nuevo, el problema del hambre no es de producción de alimentos (se produce suficiente para alimentar a todos los habitantes del Planeta) sino de distribución o de "reparto de la tarta". Véase (Vivas, 2010). 
Por eso puede decirse que la actual crisis global es mucho más dificil de gestionar que la del 29: tiene muchos más componentes y todos interconectados, con el agravante de que también ahora la desorientación ideológica y el descrédito de las democracias pueden hacer aparecer caudillos de ideología simple, si no simplista, embarcar a su país en episodios violentos frente a otros, con la diferencia, y esta es muy importante, de que ahora las potencias nucleares son varias y, en aquel tiempo, ninguna. Además de los Estados Unidos, Francia, Reino Unidos, Rusia y la China (la "banda de los cinco" en el Consejo de Seguridad de la ONU), también disponen del arma la India, Pakistán, Israel y Corea del Norte. De usarse, dejaria la crisis medioambiental en juego de niños. Como reconoce el Nuclear Posture Review Report de abril de 2010, "la amenaza de una guerra nuclear global es remota, pero el riesgo de un ataque nuclear ha aumentado" 19 .

La gravedad de la crisis (sea cual sea el perfil que adopte una posible recuperación sólo económica) es tal que son comprensibles las propuestas para cambiar las reglas del juego si se quiere mantener el statu quo ${ }^{20}$ o cambiarlas para que todo cambie efectivamente. Comenzando en el ámbito mundial, se proponen cambios en el modo de gestionar los organismos internacionales, en la manera de recibir el impacto de las actividades de multinacionales y paraísos fiscales, en particular lo referente, una vez más, a la especulación y a la irracionalidad de las burbujas especulativa y en el nivel de regulación de actividades económicas locales.

19 Departamento de Defensa, Nuclear Posture Review Report, abril de 2010 (en linea) http://www.defense.gov/npr/docs/2010\%20Nuclear\%20Posture\%20Re view\%20Report.pdf, lo cual no disminuye el riesgo de una confrontación entre la India y Pakistán, de efectos catastróficos para el Planeta, riesgo juzgado importante, para 2010, en Foreign Policy (en línea) http:/ / eurasia.foreignpolicy.com/posts/2010/01/14/top_risk_no_8_in dia_pakistan 20 "Se vogliamo che tutto rimanga com'è, bisogna che tutto cambi", que dirá Tancredi en El Gatopardo. 
Efectivamente, "otro mundo es posible", lo cual no lo hace más probable de forma necesaria. Más bien parece improbable, como improbable parecía el cambio durante la Guerra Fría. Pero el caso ahora es que no hay grupos sociales con capacidad para llevar adelante esas propuestas que, además, cuentan con la oposición frontal de aquellos que obtienen notables beneficios a partir del funcionamiento del sistema realmente existente.

La alternativa, para otros, es retirarse al pequeño grupo, retornar a la Naturaleza, consumir y vivir despacio, practicar el trueque y abandonar el dinero "oficial" y demás elementos alternativos que recuerdan a los Wandervögel de la Alemania pre-nazi, también alternativos, pero que acabaron siendo muchos de ellos miembros de Partido Nacionalsocialista. La esperanza ahora es que no vuelva a suceder algo parecido. Pero cruzarse de brazos tal vez no sea la mejor politica.

\section{Perspectivas de futuro}

Entramos, así, en una era de inestabilidad, en una "terra incognita" para la que no hay mapas y en la que las potencias que pudieron dirigir el curso de los acontecimientos para que encajasen con los intereses de sus élites ya no pueden ejercer ese poder. Filósofos como Peter Sloterdijk o Slavoj Zizek plantean que la misma idea de futuro está en crisis en las actuales circunstancias ${ }^{21}$, en antítesis a lo formulado por Paul Eluard a poco tiempo de la II Guerra Mundial, citado al comienzo del presente trabajo.

Abandonando el argumento empírico hasta ahora utilizado y pasando al argumento de autoridad, vayan algunas citas que describen las características de esa era.

21 "Des idées-force pour éviter les impasses de la globalisation. Le faceà-face Peter Sloterdijk Slavoj Zizek", Le Monde, 27 de mayo de 2011 (en linea) http://www.lemonde.fr/idees/article/2011/05/27/des-ideesforce-pour-eviter-les-impasses-de-laglobalisation_1528141_3232.html\#ens_id=1528208) 
La primera es de Paul Krugman a propósito, como titula, de la "era de la inestabilidad" en la que nos encontramos ${ }^{22}$.

"El levantar acta del fracaso de la política económica a lo largo de los últimos tres años me ha llevado a sospechar que el régimen de política económica moderada [...] es inestable inherentemente. Podría durar una generación o dos, aunque no más. Y no me estoy refiriendo a la inestabilidad financiera: la inestabilidad intelectual $\mathrm{y}$ politica son igualmente cruciales".

La segunda es de Joseph Stiglitz (2010). La inestabilidad intelectual y política a la que se refiere Krugman se podria relacionar con la situación de profunda incertidumbre. Dice así:

"La crisis ha empujado al mundo hacia una terra incognita preñada de incertidumbres. Pero hay una cosa de la que podamos estar absolutamente seguros: si los países industriales avanzados siguen por el camino que parecen haber emprendido hoy, la probabilidad de que volvamos a tener algún día no lejano una economía robusta es nula; las posiciones económicas y politicas relativas de Europa y Norteamérica quedarán muy disminuidas, así como nuestra capacidad para enfrentarnos a cuestiones de largo plazo de las que depende crucialmente nuestro futuro bienestar".

La tercera es de Immanuel Wallerstein en uno de sus comentarios $^{23}$ en este caso a propósito del viento del cambio simbolizado por la "primavera árabe":

22 Paul Krugman, "The era of instability: Where we went wrong", Truthout, 28 de diciembre de 2010, originariamente en http://www.truth-out.org/the-era-instability-where-we-wentwrong66319, inaccesible el 26 de mayo de 2011, pero disponible en dicha fecha en http://groups.google.com/group/talk.politics.misc/browse_thread/thre ad/dd3769b945654138

${ }^{23}$ Immanuel Wallerstein, "The Wind of Change - in the Arab World and Beyond", Commentary, $\mathrm{n}^{\circ} 300,1^{\circ}$ de marzo de 2011 (en línea) http://fbc.binghamton.edu/commentr.htm . En un Comentario 
"No hace falta decir que los Estados Unidos y la Europa occidental están intentando hacer todo lo que pueden para canalizar, limitar y redirigir el viento del cambio. Pero su poder ya no es el que era. Y el viento del cambio está soplando también dentro de su propio terreno. Así son los vientos. Su dirección e impulso no son constantes y, por tanto, no son predecibles. Esta vez el viento es muy fuerte. Y puede que ya no sea tan fácil canalizarlo, limitarlo y redirigirlo".

Una más, esta vez atribuida a Eduardo Galeano:

"La utopía está en el horizonte. Me acerco dos pasos, ella se aleja dos pasos. Camino diez pasos y el horizonte se desplaza diez pasos más allá. Por mucho que camine, nunca la alcanzaré. Entonces, ¿para qué sirve la utopía? Para eso: sirve para caminar".

En otras palabras, la inestabilidad y la inseguridad en el sistema mundial hacen que este se encuentre muy alejado del equilibrio. A diferencia de los sistemas en equilibrio como fue el de la Guerra Fría (y aun así cambió), el actual necesita un aporte relativamente pequeño para que se produzca un cambio. Quiere decirse con ello que las alternativas no sólo tienen sentido cuando se ven los componentes de la crisis global, sino que su impacto puede ser mucho mayor del que tendrian en condiciones de mayor equilibrio y estabilidad (y “certezas"). Esa es la función de la utopía (alternativa).

Pero todavia hay clases, como las había en la Guerra Fría. No sólo hay que buscar y elaborar las alternativas, sino que hay que encontrar los portadores de las mismas, los actores sociales que las lleven adelante, sean "los de arriba", "los de abajo" o "los de en medio" o "indignados". También hace falta, si se quiere ir más allá de la retórica, determinar sus condiciones de posibilidad y los medios necesarios para que

posterior ('The Political Quandary of Barack Obama", Commentary, $\mathrm{n}^{\mathrm{o}}$ 306, $1^{\circ}$ de junio de 2011) añadirá: "The world is moving on at a rapid pace. In a world with so many uncertainties and unpredictable actors, the most dangerous "loose gun" is turning out to be the United States". 
sean efectivas. La cuestión no es únicamente el cambio (que siempre se produce) sino el sentido que tome el mismo y a favor de quiénes se produzca. Y esa es, probablemente, la tarea más difícil.

Manuel Monereo (2011) lo planteaba de la siguiente forma:

"No hay soluciones fáciles y cualquiera de ellas va a exigir compromiso, organización y movilización social. La condición fundamental para que las y los de abajo influyan $\mathrm{y}$ tengan $\mathrm{voz}$ es crear un auténtico poder ciudadano. Hay que indignarse, rebelarse y, sobre todo, luchar. El primer derecho a reivindicar es el derecho a resistirse a la tiranía de la oligarquía financiera y a proteger nuestra sociedad del capitalismo. La auténtica alternativa está en optar o por la regresión social y la involución autoritaria o por una sociedad justa, democrática y fraterna, es decir, una república de hombres y mujeres libres e iguales.

Para organizar la resistencia es necesario, en primer lugar, tomar la realidad tal como es y no engañar ni engañarse [Énfasis añadido JMT]. La crisis no ha hecho nada más que comenzar y durará mucho tiempo. El tiempo pasado no volverá y juntos tenemos que construir nuestro futuro".

No hay fin de la Historia. Siempre habrá contradicciones y, por tanto, alternativas. Pero, por lo dicho, son particularmente necesarias en la actual coyuntura catastrófica y podrían ser particularmente eficaces dado el estado de desequilibrio del sistema.

\section{REFERENCIAS}

Acosta, A, (2009): La maldición de la abundancia, Quito, Abya-Yala.

Acosta, A. (2011): "Ecuador, de la trampa petrolera a la minera", en A. Acosta y otros, Colonialismos del siglo XXI. Negocios extractivos y defensa del territorio en América Latina, Barcelona, Icaria, 2011, págs. 37-73. 
Amalrik, A. (1970): Will the Soviet Union survive until 1984?, Nueva York, Harper and Row.

Arrighi, G. y Silver, B. (2001): Caos y orden en el sistemamundo moderno, Madrid, Akal.

Bailey, R. (2011): Cultivar un mundo mejor. Justicia alimentaria en un mundo con recursos limitados, Oxfam Internacional (en

linea) http://firgoa.usc.es/drupal/files/Oxfam_Cultivar_un_fu turo_mejor.pdf, acceso el 18 de julio de 2011.

Brown, L.R (2011): "The New Geopolitics of Food", Foreign Policy, mayo/junio (en linea) http://www.foreignpolicy.com/articles/2011/04/25/the _new_geopolitics_of_food?page $=0,0$, acces el 18 de julio de 2011.

CEPAL (2010): La República Popular China y América Latina y el Caribe: hacia una relación estratégica, mayo de 2010 (en

linea)

http://www.cepal.org/publicaciones/xml/2/39082/RP

China_America_Latina_el_Caribe_una_relacion_estrategi ca_906.pdf, acceso el 18 de julio de 2011.

Chomsky, N. (2010): “China's Growing Independence and the New World Order", In These Times, 5 de octubre de 2010 (en

linea)

http://www.inthesetimes.com/article/6499/chinas_gro wing_independence_and_the_new_world_order/ acceso el 18 de julio de 2011 .

Chomsky, N. y Herman, E.S. (2000): Los guardianes de la libertad, Barcelona, Crítica.

Ehrlich, A. (1984): "Nuclear Winter", Bulletin of the Atomic Scientists, XL, 4. Special Suplement.

Fondo Monetario Internacional (2011): Perspectivas de la economia mundial. Abril 2011. Las tensiones de una recuperación a dos velocidades (en línea) http://www.imf.org/external/spanish/pubs/ft/weo/20 $11 / 01 / \mathrm{pdf} /$ texts.pdf, acceso el 18 de julio de 2011 .

Foreman, A, (2011): A World on Fire, Nueva York, Random House.

Friedrichs, J. (2010): "Global energy crunch: How different parts of the world would react to a peak oil scenario", Energy Policy, 38, 8: 4.562-4.569 (en linea) 
http://www.qeh.ox.ac.uk/pdf/pdf-

research/Global\%20Energy\%20Crunch.pdf, acceso el 18 de julio de 2011.

Galtung, J. (1971): "A structural theory of imperialism", Journal of Peace Research, VIII, 2: 81-117.

Galtung, J. (1985): Hitlerismo, stalinismo y reaganismo: tres variaciones sobre un tema de Orwell, Alicante, Instituto de Estudios Juan Gil-Albert.

Galtung, J. (2010): La caida del Imperio de los EE.UU. ¿Y luego... qué?, México, Transcend University Press.

Goldstein, R.J. (2001): Political repression in modern America: From 1870 to 1976, Chicago, University of Illinois Press.

Kennedy, P.M. (2004): Auge y caida de las grandes potencias, Barcelona, Plaza\&Janés.

Lenin,V.I. (1918): "Letter to American Workers", publicada originalmente en Pravda, No. 178, 22 de agosto de 1918, traducida

al inglés, en http://www.marxists.org/archive/lenin/works/1918/a ug/20.htm, acceso el 18 de julio de 2011.

Lovelock, J. (2009): The vanishing face of Gaia: A final warning, Nueva York, Basic Books.

Michel, S. y Beuret, M. (2009): China Safari. On the trial of Beijing's expansion in Africa, Nueva York, Nation Books.

Monereo, M. (2011): "Por la revolución ciudadana", El viejo topo, 20 de mayo de 2011 (en línea) http://www.elviejotopo.com/web/otrostextos.php, acceso el 18 de julio de 2011.

Oliveres, A. (2010): Aturem la crisi. Les perversions d'un sistema que és possible canviar, Barcelona, Angle Editorial.

Perkins, J. (2005): Confesiones de un sicario económico, Buenos Aires, Plume.

Scruton, R. (2010): Usos del pesimismo. El peligro de la falsa esperanza, Barcelona, Ariel.

Smith, P.B. y Max-Neef M. (2011), Economics Unmasked, Totnes, Devon, Green Books.

Stiglitz, J. (20102): Freefall: Free Markets and the Sinking of the Global Economy, Londres, Penguin.

Stiglitz, J.E. (2011): "La crisis ideológica del capitalismo occidental", Project Syndicate, 6 de julio de 2011, (en 
linea)

http://www.projectsyndicate.org/commentary/stiglitz140/Spanish, acceso el 18 de julio de 2011.

The International Workingmen's Association (1865): Address of the International Working Men's Association to Abraham Lincoln, President of the United States of America. Presented to U.S. Ambassador Charles Francis Adams, 28 de enero de 1865 (en línea) http://www.marxists.org/archive/marx/iwma/docume nts/1864/lincoln-letter.htm, acceso el 18 de julio de 2011.

Touraine, A. (2011): Después de la crisis. Por un futuro sin marginación, Barcelona, Paidós.

Vidal-Beneyto, J. (2010): La corrupción de la democracia, Madrid, Catarata, 2010.

Vivas, E. (2010): "Soberanía alimentaria: podemos alimentar al mundo", ALAI - América Latina en movimiento, 30 de marzo de 2010 (en linea) http://alainet.org/active/37065, acceso el 18 de julio de 2011 ,

Wallerstein, I. (2005a): "El fin de las certidumbres y los intelectuales comprometidos", Disenso, 47.

Wallerstein, I. (2005b): La crisis estructural del capitalismo, México, Los libros de Contrahistorias.

Wallerstein, I. (2005c): "Death by a thousand cuts", Commentary, $\mathrm{N}^{\circ} 160,1^{\circ}$ de mayo de 2005 (en linea) fbc.binghamton.edu/commentr.htm, acceso el 18 de julio de 2011.

Welzer, H, (2011): Guerras climáticas. Por qué mataremos (y nos matarán) en el siglo XXI, Barcelona, Icaria.

\section{FORMA DE CITAR ESTE TRABAJO EN BIBLIOGRAFÍAS HOW TO CITE THIS ARTICLE IN BIBLIOGRAPHIES}

Tortosa, J. M. (2011): “Antecedentes y viabilidad de alternativas en el actual sistema-mundo", Revista Latina de Sociologia, $\mathrm{n}^{\mathrm{o}}$ 1: 224-257, http://revistalatinadesociologia.com, ISSN 2253-6469 